\title{
ASYMPTOTIC BEHAVIOR OF A CLASS OF NONLINEAR DIFFERENCE EQUATIONS
}

STEVO STEVIĆ

Received 7 April 2006; Accepted 28 May 2006

Motivated by some results of L. Berg (2002), in this paper we find the second member in the asymptotic development of some of the positive solutions of a class of difference equations of second and third orders. The main result in this paper partially solves an open problem by S. Stević (2003), and it is applied to some classes of mathematical biology models, for example, generalized Beverton-Holt stock recruitment model, flour beetle population model, mosquito population equations, and discrete delay logistic difference equation.

Copyright (c) 2006 Stevo Stević. This is an open access article distributed under the Creative Commons Attribution License, which permits unrestricted use, distribution, and reproduction in any medium, provided the original work is properly cited.

\section{Introduction}

Recently there has been a great interest in studying nonlinear difference equations and systems (cf. [1-36] and the references therein). One of the reasons for this is a necessity for some techniques which can be used in investigating equations arising in mathematical models describing real-life situations in population biology, economy, probability theory, genetics, psychology, sociology, and so forth. Such equations also appear naturally as discrete analogues of differential equations which model various biological and economical systems (see, e.g, [7, 13, 16-18, 20, 24, 28, 29] and the references therein).

There are sequences defined by recurrence formulae such that we know their asymptotic behavior, see, for example, $[11,12,14,15,19,21,23,29,30,32,33,36]$, that is, we know the first member in their asymptotic behavior.

The following theorem was established in [21, Problem I, page 174]. The proof appearing there is attributed to Jacobsthal.

Theorem 1.1. Let $f:(0, \alpha) \rightarrow(0, \alpha)$, where $\alpha>0$, be a continuous function such that $0<$ $f(x)<x$ for every $x \in(0, \alpha)$ and $f(x)=x-a x^{k}+b x^{k+p}+o\left(x^{k+p}\right)$, when $x \rightarrow+0$, where 
2 Asymptotic behavior of some difference equations

$k>1, p, a$, and $b$ are positive numbers. Let $x_{0} \in(0, \alpha)$ and $x_{n}=f\left(x_{n-1}\right), n \geq 1$. Then

$$
x_{n} \sim \frac{1}{((k-1) a n)^{1 /(k-1)}} .
$$

Our version of this proof is somewhat different to its original form, and the idea and structure of this version was the starting point and inspiration for our further investigations, see [23]. In [24] we have noted, by an example, that there are sequences defined by difference equations of second order which behave similarly to those sequences in Theorem 1.1. Motivated by the example in [29], we have found the asymptotics of all positive solutions of a nonlinear difference equation of second order.

A natural problem was whether Theorem 1.1 could be extended in the case of difference equations of order greater than one. The problem was not only of great importance in its own right. This is important because there is a large class of mathematical biology models which are described by equations of this type. For example,

discrete delay logistic difference equation [20]:

$$
N_{t+1}=\frac{\alpha N_{t}}{1+\beta N_{t-k}}, \quad \alpha, \beta>0, k \in \mathbb{N}
$$

generalized Beverton-Holt stock recruitment model [7, 13]:

$$
x_{n+1}=a x_{n}+\frac{b x_{n-1}}{1+c x_{n-1}+d x_{n}}, \quad x_{0}, x_{1}>0, n=1,2,3, \ldots,
$$

where $a \in(0,1), b \in \mathbb{R}_{+}$, and $c, d \in \mathbb{R}_{+} \cup\{0\}$, with $c+d>0$;

flour beetle population model [18]:

$$
x_{n+3}=a x_{n+2}+b x_{n} e^{-\left(c x_{n+2}+d x_{n}\right)}, \quad n=0,1,2, \ldots,
$$

with $a, b, c, d \geq 0$ and $c+d>0$.

mosquito population equations [16]:

$$
x_{n+1}=\left(a x_{n}+b x_{n-1} e^{-x_{n-1}}\right) e^{-x_{n}}, \quad x_{0}, x_{1}>0, n=1,2,3, \ldots,
$$

where $a \in(0,1), b \in[0, \infty)$, and

$$
x_{n+1}=\left(\alpha x_{n}+\beta x_{n-1}\right) e^{-x_{n}}, \quad x_{0}, x_{1}>0, n=1,2,3, \ldots,
$$

where $\alpha \in[0,1), \beta \in(0, \infty)$.

One of the most interesting cases of these equations is when the sum of the main coefficients of the equations is equal to 1 , that is, when $\alpha=1$ for (1.2), $a+b=1$ for (1.3), (1.4), and (1.5), and $\alpha+\beta=1$ for (1.6).

A natural question is to find the first term in the asymptotic behavior of the solutions of (1.3) $-(1.5)$ for $a+b=1$ and of (1.6) when $\alpha+\beta=1$.

In $[29,32]$ we have generalized Theorem 1.1 in the case of sequences defined by a difference equations of order more than one, where among other results we completely described the asymptotic behavior of solutions of (1.3)-(1.6) in these cases. 
In [32] we have proved the following theorem.

Theorem 1.2. Let $f:[0,+\infty)^{k} \rightarrow[0,+\infty)$ be a continuous function such that

(a) $0<f\left(z_{1}, \ldots, z_{k}\right)<\max \left\{z_{1}, \ldots, z_{k}\right\}$ for all $z_{1}, \ldots, z_{k} \in[0, \alpha)$, such that $\left(z_{1}, z_{2}, \ldots, z_{k}\right) \neq$ $(0,0, \ldots, 0)$, where $\alpha \in(0, \infty]$ and $k \in \mathbb{N}$;

(b) $f\left(z_{1}, \ldots, z_{k}\right)=p_{1} z_{1}+\cdots+p_{k} z_{k}-\sum_{s=m}^{+\infty} K_{s}\left(z_{1}, \ldots, z_{k}\right)$ uniformly as $z_{1}^{2}+\cdots+z_{k}^{2} \rightarrow$ 0 , where $m>1, p_{1}, \ldots, p_{k} \in[0,1)$, and $K_{s}\left(z_{1}, \ldots, z_{k}\right)$ is a homogeneous polynomial of order $s$;

(c) $\sum_{i=1}^{k} p_{i}=1$;

(d) $f\left(z_{1}, \ldots, z_{k}\right)$ is nondecreasing in each variable in a neighborhood of the origin;

(e) $K_{m}(1, \ldots, 1)>0$.

Then the sequence defined by

$$
x_{n+1}=f\left(x_{n}, \ldots, x_{n-k+1}\right)
$$

with initial conditions $x_{0}, x_{1}, \ldots, x_{k-1} \in[0, \alpha)$ satisfies the following asymptotic formula:

$$
x_{n} \sim\left(\frac{P_{k}^{\prime}(1)}{(m-1) K_{m}(1, \ldots, 1)}\right)^{1 /(m-1)} \frac{1}{\sqrt[m-1]{n}},
$$

where $P_{k}(\lambda)=\lambda^{k}-p_{1} \lambda^{k-1}-\cdots-p_{k-1} \lambda-p_{k}$.

Remark 1.3. From the proof of Theorem 1.2, it is easy to see that condition (b) can be replaced by the following condition:

$$
(b)^{\prime} f\left(z_{1}, \ldots, z_{k}\right)=p_{1} z_{1}+\cdots+p_{k} z_{k}-K_{m}\left(z_{1}, \ldots, z_{k}\right)+o\left(\left(z_{1}^{2}+\cdots+z_{k}^{2}\right)^{m / 2}\right),
$$

uniformly as $z_{1}^{2}+\cdots+z_{k}^{2} \rightarrow 0$, where $m>1, p_{1}, \ldots, p_{k} \in[0,1)$, and $K_{m}\left(z_{1}, \ldots, z_{k}\right)$ is a homogeneous polynomial of order $m$.

Remark 1.4. Note that if $p_{1}, \ldots, p_{k} \in(0,1)$, then condition (d) is automatically satisfied, since the partial derivatives of the function $f$ are positive in a neighborhood of the origin.

The convergence of the sequences in Theorem 1.2 follows, for example, from some results, in [25, 27]. For closely related results, see also [1, 8-10, 22, 24-28, 31].

Applying Theorem 1.2 to (1.3)-(1.6), we obtain that the following asymptotics hold.

Equation (1.3). If $a, b \in(0,1)$ and $a+b=1$, then

$$
x_{n} \sim \frac{2-a}{(1-a)(c+d) n} .
$$

Equation (1.4). If $a, b \in(0,1)$ and $a+b=1$, then

$$
x_{n} \sim \frac{3-2 a}{(1-a)(c+d) n} .
$$

Equation (1.5). If $a, b \in(0,1)$ and $a+b=1$, then

$$
x_{n} \sim \frac{1}{n} .
$$


4 Asymptotic behavior of some difference equations

Equation (1.6). If $\alpha, \beta \in(0,1)$ and $\alpha+\beta=1$, then

$$
x_{n} \sim \frac{2-\alpha}{n} .
$$

For the class of difference equations of first order defined in Theorem 1.1, we described in [23], under some additional conditions, a method for finding the next members in asymptotic developments of its solutions. It is a natural question to find the other members in the asymptotic development of the solutions of (1.3)-(1.6). Hence in [32] we have offered the following problem.

Open problem 1.5. Find the second member in the asymptotic development of the solutions of (1.3)-(1.6), that is, find the sequence $y_{n}$ such that

$$
\lim _{n \rightarrow \infty} \frac{x_{n}-a / n}{y_{n}}=1
$$

The main object in the paper is to find the second member in the asymptotic development of some of the positive solutions of a class of nonlinear difference equations generalizing (1.2)-(1.6).

\section{The inclusion theorem}

In order to prove the open problem, we need the following result.

THEOREM 2.1. Let $f: I^{k} \rightarrow I$ be a continuous and nondecreasing function in each argument on the interval $I \subseteq \mathbb{R}$, and let $\left(y_{n}\right)$ and $\left(z_{n}\right)$ be sequences in $I$, with $y_{n}<z_{n}$ for $n \geq n_{0}$ and such that

$$
y_{n+1} \leq f\left(y_{n}, \ldots, y_{n-k+1}\right), \quad f\left(z_{n}, \ldots, z_{n-k+1}\right) \leq z_{n+1}, \quad n \geq n_{0}+k-1 .
$$

Let further $x_{n}$ be a solution of the following difference equation:

$$
x_{n+1}=f\left(x_{n}, \ldots, x_{n-k+1}\right)
$$

such that

$$
y_{n} \leq x_{n} \leq z_{n}, \quad \text { for } n \in\left\{n_{0}, \ldots, n_{0}+k-1\right\},
$$

then

$$
y_{n} \leq x_{n} \leq z_{n}, \quad \text { for } n \geq n_{0} .
$$

Proof. We prove the theorem by induction. If $n=n_{0}+k$, then using (2.1) and the monotonicity of the function $f$ we have

$$
y_{n_{0}+k} \leq f\left(y_{n_{0}+k-1}, \ldots, y_{n_{0}}\right) \leq f\left(x_{n_{0}+k-1}, \ldots, x_{n_{0}}\right) \leq f\left(z_{n_{0}+k-1}, \ldots, z_{n_{0}}\right) \leq z_{n_{0}+k},
$$

that is,

$$
y_{n_{0}+k} \leq x_{n_{0}+k} \leq z_{n_{0}+k} .
$$


Assume now that it has been proved that

$$
y_{n} \leq x_{n} \leq z_{n}, \quad \text { for } n \in\left\{n_{0}, \ldots, m\right\},
$$

where $m \geq n_{0}+k$, then using again (2.1), induction hypothesis, and the monotonicity of the function $f$ we have

$$
y_{m+1} \leq f\left(y_{m}, \ldots, y_{m-k+1}\right) \leq f\left(x_{m}, \ldots, x_{m-k+1}\right) \leq f\left(z_{m}, \ldots, z_{m-k+1}\right) \leq z_{m+1},
$$

that is, $y_{m+1} \leq x_{m+1} \leq z_{m+1}$, finishing the proof.

Remark 2.2. Some other results of this type and their applications can be found, for example, in $[2-6,34,35]$.

Remark 2.3. It suffices that the hypotheses concerning $f$ are satisfied in the strip (2.4).

\section{Main result}

In this section, we formulate and prove the main result in this paper. As a consequence of this result, we find the second member in the asymptotics of some positive solutions of (1.3)-(1.6).

Theorem 3.1. Consider (1.7), where $k \in\{2,3\}$. Then the following statements are true.

(a) For $k=2$ and $p \in(0,1)$, there is a positive solution of (1.7) with the following asymptotics:

$$
x_{n}=\frac{2-p}{K_{2}(1,1) n}+b \frac{\ln n}{n^{2}}+o\left(\frac{\ln n}{n^{2}}\right)
$$

as $n \rightarrow \infty$, where

$$
f(x, y)=p x+(1-p) y-K_{2}(x, y)-K_{3}(x, y)+o\left(\left(x^{2}+y^{2}\right)^{3 / 2}\right),
$$

as $x^{2}+y^{2} \rightarrow 0$

$$
b=\frac{p(s-q)-2(r+2 s)}{K_{2}(1,1)^{2}}-\frac{K_{3}(1,1)}{K_{2}(1,1)^{3}}(2-p)^{2},
$$

$K_{2}(x, y)=q x^{2}+r x y+s y^{2}$ is a positive definite form, and $K_{3}(x, y)$ is a homogeneous polynomial in variables $x$ and $y$ of third order.

(b) For $k=3$ and $p, q, p+q \in(0,1)$, there is a positive solution of (1.7) with the following asymptotics:

$$
x_{n}=\frac{3-q-2 p}{K_{2}(1,1,1) n}+b \frac{\ln n}{n^{2}}+o\left(\frac{\ln n}{n^{2}}\right)
$$

as $n \rightarrow \infty$, where

$$
f(x, y, z)=p x+q y+(1-p-q) z-K_{2}(x, y, z)-K_{3}(x, y, z)+o\left(\left(x^{2}+y^{2}+z^{2}\right)^{3 / 2}\right),
$$


6 Asymptotic behavior of some difference equations

$$
\begin{aligned}
& \text { as } x^{2}+y^{2}+z^{2} \rightarrow 0, \\
& b=\frac{3(r-3 t-s-v-2 w)-q(3 r+s+2 u+v-t)-2 p(2 r-2 t+u-w)}{K_{2}(1,1,1)^{2}} \\
& \quad+\frac{K_{3}(1,1,1)}{K_{2}(1,1,1)^{3}}(3-2 p-q)^{2},
\end{aligned}
$$

$K_{2}(x, y, z)=r x^{2}+s y^{2}+t z^{2}+u x y+v x z+w y z$ is a positive definite form, and $K_{3}(x, y)$ is a homogeneous polynomial in variables $x, y$, and $z$ of third order.

Proof. In both cases, we suppose that there are solutions which have the following asymptotics:

$$
x_{n}=\frac{a}{n}+\frac{b \ln n}{n^{2}}+o\left(\frac{\ln n}{n^{2}}\right)=\varphi_{n}+o\left(\frac{\ln n}{n^{2}}\right)
$$

as $n \rightarrow \infty$.

We show this by finding the values of the coefficients $a$ and $b$.

(a) Let

$$
F(x, y, z)=x-f(y, z)
$$

Choosing $a=(2-p) /(q+r+s)=(2-p) /\left(K_{2}(1,1)\right)$ and $b$ arbitrary, comparing coefficients in

$$
F\left(\varphi_{n+1}, \varphi_{n}, \varphi_{n-1}\right)=0
$$

by some calculations, we obtain

$$
F\left(\varphi_{n+1}, \varphi_{n}, \varphi_{n-1}\right) \sim \frac{(2-p)\left(b-b_{0}\right)}{n^{3}}
$$

where

$$
b_{0}=\frac{p(s-q)-2(r+2 s)}{K_{2}(1,1)^{2}}-\frac{K_{3}(1,1)}{K_{2}(1,1)^{3}}(2-p)^{2} .
$$

Let $b_{1}$ and $b_{2}$ be such that $b_{1}>b_{0}$ and $b_{2}<b_{0}$. With the notations

$$
\begin{aligned}
& y_{n}=\frac{a}{n}+\frac{b_{2} \ln n}{n^{2}}, \\
& z_{n}=\frac{a}{n}+\frac{b_{1} \ln n}{n^{2}}
\end{aligned}
$$

and from (3.10), we get

$$
\begin{aligned}
& F\left(y_{n+1}, y_{n}, y_{n-1}\right) \sim \frac{(2-p)\left(b_{2}-b_{0}\right)}{n^{3}}<0, \\
& F\left(z_{n+1}, z_{n}, z_{n-1}\right) \sim \frac{(2-p)\left(b_{1}-b_{0}\right)}{n^{3}}>0 .
\end{aligned}
$$


These relations show that inequalities (2.1) are satisfied for sufficiently large $n$, where $f$ is defined in Theorem 3.1(a) and $F$ is given by (3.8). Thus, since for sufficiently large $n$ we can chose $b$ arbitrary close to $b_{0}$, in view of Theorem 2.1 , it follows that in this case there is a solution of (1.7) which has asymptotics (3.4).

(b) Let

$$
F(x, y, z, w)=x-f(y, z, w) .
$$

Choosing $a=(3-q-2 p) /\left(K_{2}(1,1,1)\right)$ and $b$ arbitrary, comparing coefficients in

$$
F\left(\varphi_{n+1}, \varphi_{n}, \varphi_{n-1}, \varphi_{n-2}\right)=0,
$$

the DERIVE system yields

$$
F\left(\varphi_{n+1}, \varphi_{n}, \varphi_{n-1}, \varphi_{n-2}\right) \sim \frac{(3-q-2 p)\left(b-b_{0}\right)}{n^{3}},
$$

where

$$
\begin{aligned}
b_{0}= & \frac{3(r-3 t-s-v-2 w)-q(3 r+s+2 u+v-t)-2 p(2 r-2 t+u-w)}{K_{2}(1,1,1)^{2}} \\
& +\frac{K_{3}(1,1,1)}{K_{2}(1,1,1)^{3}}(3-2 p-q)^{2} .
\end{aligned}
$$

Let $b_{1}$ and $b_{2}$ be such that $b_{1}>b_{0}$ and $b_{2}<b_{0}$. With the notations (3.12) and from (3.16), we get

$$
\begin{aligned}
& F\left(y_{n+1}, y_{n}, y_{n-1}, y_{n-2}\right) \sim \frac{(3-q-2 p)\left(b_{2}-b_{0}\right)}{n^{3}}<0, \\
& F\left(z_{n+1}, z_{n}, z_{n-1}, z_{n-2}\right) \sim \frac{(3-q-2 p)\left(b_{1}-b_{0}\right)}{n^{3}}>0 .
\end{aligned}
$$

These relations show that inequalities (2.1) are satisfied for sufficiently large $n$, where $f$ is given in Theorem 3.1(b) and $F$ is given by (3.14). Applying Theorem 2.1, we obtain that in this case there is a solution of (1.7) which has asymptotics (3.4).

3.1. Case of discrete delay logistic difference equation. In the case of a general $k$, we only consider the discrete delay logistic difference equation (1.2) with $\alpha=1$, that is,

$$
x_{n+1}=\frac{x_{n}}{1+\beta x_{n-k}} .
$$

From [29, Theorem 2], it follows that

$$
x_{n} \sim \frac{1}{\beta n} .
$$

Let

$$
F(x, u, v)=x-\frac{u}{1+\beta v} .
$$


8 Asymptotic behavior of some difference equations

We assume that (3.19) has a positive solution $x_{n}$ with the asymptotics

$$
x_{n}=\frac{1}{n}+b \frac{\ln n}{n^{2}}+o\left(\frac{\ln n}{n^{2}}\right)
$$

and also that $\beta=1$, otherwise we consider the sequence $y_{n}=\beta x_{n}$. Let

$$
\varphi_{n}=\frac{1}{n}+b \frac{\ln n}{n^{2}} .
$$

Then by well-known asymptotics formulae, we have

$$
\begin{aligned}
F\left(\varphi_{n+1}, \varphi_{n}, \varphi_{n-k}\right)= & \varphi_{n+1}-\varphi_{n}+\varphi_{n} \varphi_{n-k}-\varphi_{n} \varphi_{n-k}^{2}+o\left(\frac{1}{n^{3}}\right) \\
= & \frac{1}{n}\left(1+\frac{1}{n}\right)^{-1}+b \frac{\ln n}{n^{2}}\left(1+\frac{1}{n}\right)^{-2}+b \frac{\ln (1+1 / n)}{n^{2}}\left(1+\frac{1}{n}\right)^{-2}-\frac{1}{n}-b \frac{\ln n}{n^{2}} \\
& +\left(\frac{1}{n}+b \frac{\ln n}{n^{2}}\right)\left(\frac{1}{n}\left(1-\frac{k}{n}\right)^{-1}+b \frac{\ln n+\ln (1-k / n)}{n^{2}}\left(1-\frac{k}{n}\right)^{-2}\right) \\
& -\left(\frac{1}{n}+b \frac{\ln n}{n^{2}}\right)\left(\frac{1}{n}\left(1-\frac{k}{n}\right)^{-1}+b \frac{\ln n+\ln (1-k / n)}{n^{2}}\left(1-\frac{k}{n}\right)^{-2}\right)^{2}+o\left(\frac{1}{n^{3}}\right) \\
= & \frac{1}{n}-\frac{1}{n^{2}}+\frac{1}{n^{3}}+b \frac{\ln n}{n^{2}}-2 b \frac{\ln n}{n^{3}}+\frac{b}{n^{3}}-\frac{1}{n}-b \frac{\ln n}{n^{2}} \\
& +\frac{1}{n^{2}}+2 b \frac{\ln n}{n^{3}}+\frac{k}{n^{3}}-\frac{1}{n^{3}}+o\left(\frac{1}{n^{3}}\right) \sim \frac{b+k}{n^{3}} .
\end{aligned}
$$

Hence, in this case, we have that $b=-k$ (which implies that $b=-k / \beta$ if $\beta \neq 1$ ) in (3.21). Denote $b_{0}=-k / \beta$. Using (3.12) and choosing $b_{1}$ and $b_{2}$ such that $b_{1}>b_{0}$ and $b_{2}<b_{0}$, we obtain

$$
\begin{gathered}
F\left(y_{n+1}, y_{n}, y_{n-k}\right) \sim \frac{\left(b_{2}-b_{0}\right)}{n^{3}}<0, \\
F\left(z_{n+1}, z_{n}, z_{n-k}\right) \sim \frac{\left(b_{1}-b_{0}\right)}{n^{3}}>0 .
\end{gathered}
$$

Now note that from this and by Theorem 2.1 we could conclude that there is a positive solution of (3.19) with the following asymptotics:

$$
x_{n}=\frac{1}{\beta n}-\frac{k}{\beta} \frac{\ln n}{n^{2}}+o\left(\frac{\ln n}{n^{2}}\right)
$$

as $n \rightarrow \infty$, if the monotonicity conditions were not violated.

Hence, for the readers interested in this research area, we leave the following conjecture.

CONJECTURe 3.2. Shows that there is a positive solution of (3.19) with asymptotics (3.22). 
Remark 3.3. It is interesting that Conjecture 3.2 cannot be confirmed also by [4, Theorem 2.1] (see also [5]), since for the case of (3.19), two coefficients with the largest moduli in [4, formula (2.3)] have the same moduli so that [4, condition (2.4)] is not satisfied. We would like to point out that [4, Theorem 2.1] was applied with a success at many points, for example, in $[4,34]$.

\section{References}

[1] K. S. Berenhaut and E. G. Goedhart, Explicit bounds for second-order difference equations and a solution to a question of Stević, Journal of Mathematical Analysis and Applications 305 (2005), no. $1,1-10$.

[2] L. Berg, Asymptotische Darstellungen und Entwicklungen, Hochschulbücher für Mathematik, Band 66, VEB Deutscher Verlag der Wissenschaften, Berlin, 1968.

[3] _ On the asymptotics of nonlinear difference equations, Zeitschrift für Analysis und ihre Anwendungen 21 (2002), no. 4, 1061-1074.

[4] _ Inclusion theorems for non-linear difference equations with applications, Journal of Difference Equations and Applications 10 (2004), no. 4, 399-408.

[5] _ Corrections to "Inclusion theorems for non-linear difference equations with applictions", Journal of Difference Equations and Applications 11 (2005), no. 2, 181-182.

[6] L. Berg and L. von Wolfersdorf, On a class of generalized autoconvolution equations of the third kind, Zeitschrift für Analysis und ihre Anwendungen 24 (2005), no. 2, 217-250.

[7] R. J. Beverton and S. J. Holt, On the Dynamics of Exploited Fish Populations, vol. 19, Fish. Invest., London, 1957.

[8] J. Bibby, Axiomatisations of the average and a further generalisation of monotonic sequences, Glasgow Mathematical Journal 15 (1974), 63-65.

[9] D. Borwein, Convergence criteria for bounded sequences, Proceedings of the Edinburgh Mathematical Society. Series II 18 (1972/1973), 99-103.

[10] E. T. Copson, On a generalisation of monotonic sequences, Proceedings of the Edinburgh Mathematical Society. Series II 17 (1970/1971), 159-164.

[11] D. Cox, Problem E 3034, American Mathematical Monthly 91 (1984), 58.

[12] N. G. de Bruijn, Asymptotic Methods in Analysis, Bibliotheca Mathematica, vol. 4, NorthHolland, Amsterdam, 1958.

[13] R. DeVault, G. Dial, V. L. Kocic, and G. Ladas, Global behavior of solutions of $x_{n+1}=a x_{n}+$ $f\left(x_{n}, x_{n-1}\right)$, Journal of Difference Equations and Applications 3 (1998), no. 3-4, 311-330.

[14] A. Ya. Dorogovcev, Problem MS'88 no. 6, Matematika Segodnya 6 (1990), 176-177 (Russian).

[15] A. W. Goodman, Problem 6610, American Mathematical Monthly 96 (1989), 774.

[16] E. A. Grove, C. M. Kent, G. Ladas, S. Valicenti, and R. Levins, Global stability in some population models, Communications in Difference Equations. Proceedings of the 4th International Conference on Difference Equations (Poznan, 1998), Gordon and Breach, Amsterdam, 2000, pp. 149-176.

[17] H.-F. Huo and W.-T. Li, Permanence and global stability of positive solutions of a nonautonomous discrete ratio-dependent predator-prey model, Discrete Dynamics in Nature and Society 2005 (2005), no. 2, 135-144.

[18] Y. Kuang and J. M. Cushing, Global stability in a nonlinear difference-delay equation model of flour beetle population growth, Journal of Difference Equations and Applications 2 (1996), no. 1, 31-37.

[19] A. G. Pakes, Asymptotic behaviour of monotone weakly-nonlinear recurrences, The Australian Mathematical Society. Gazette 29 (2002), no. 2, 91-98.

[20] E. C. Pielou, Population and Community Ecology, Gordon and Breach, New York, 1974.

[21] G. Polya and G. Szegö, Aufgaben und lehrsätze aus der analysis, Verlag von Julius, Berlin, 1925. 


\section{Asymptotic behavior of some difference equations}

[22] D. C. Russell, On bounded sequences satisfying a linear inequality, Proceedings of the Edinburgh Mathematical Society. Series II 19 (1974/1975), 11-16.

[23] S. Stević, Asymptotic behaviour of a sequence defined by iteration, Matematichki Vesnik 48 (1996), no. 3-4, 99-105.

[24] Behavior of the positive solutions of the generalized Beddington-Holt equation, Panamerican Mathematical Journal 10 (2000), no. 4, 77-85.

[25] _ A generalization of the Copson's theorem concerning sequences which satisfy a linear inequality, Indian Journal of Mathematics 43 (2001), no. 3, 277-282.

[26] - On the recursive sequence $x_{n+1}=-\frac{1}{x_{n}}+\frac{A}{x_{n-1}}$, International Journal of Mathematics and Mathematical Sciences 27 (2001), no. 1, 1-6.

[27] _ A global convergence result, Indian Journal of Mathematics 44 (2002), no. 3, 361-368.

[28]_A global convergence results with applications to periodic solutions, Indian Journal of Pure and Applied Mathematics 33 (2002), no. 1, 45-53.

[29] - Asymptotic behavior of a sequence defined by iteration with applications, Colloquium Mathematicum 93 (2002), no. 2, 267-276.

[30] _ Asymptotic behaviour of a sequence defined by a recurrence formula. II, The Australian Mathematical Society. Gazette 29 (2002), no. 4, 209-215.

[31] (2002), no. 3, 405-414.

[32] _ Asymptotic behavior of a nonlinear difference equation, Indian Journal of Pure and Applied Mathematics 34 (2003), no. 12, 1681-1687.

[33] On the recursive sequence $x_{n+1}=x_{n}+\frac{x_{n}^{\alpha}}{n^{\beta}}$, Bulletin of the Calcutta Mathematical Society 95 (2003), no. 1, 39-46.

[34] Global stability and asymptotics of some classes of rational difference equations, Journal of Mathematical Analysis and Applications 316 (2006), no. 1, 60-68.

[35] On positive solutions of $a(k+1)$-th order difference equation, Applied Mathematics Letters 19 (2006), no. 5, 427-431.

[36] International student competition in mathematics, Belgrade 1984.

Stevo Stević: Mathematical Institute, Serbian Academy of Science and Arts, Knez Mihailova 35/I, Beograd 11000, Serbia

E-mail addresses: sstevic@ptt.yu; sstevo@matf.bg.ac.yu 


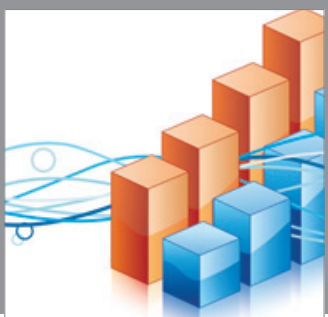

Advances in

Operations Research

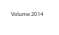

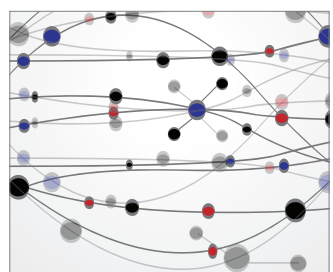

\section{The Scientific} World Journal
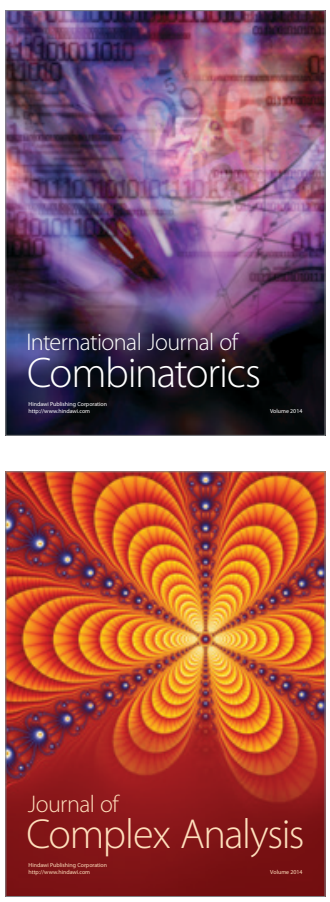

International Journal of

Mathematics and

Mathematical

Sciences
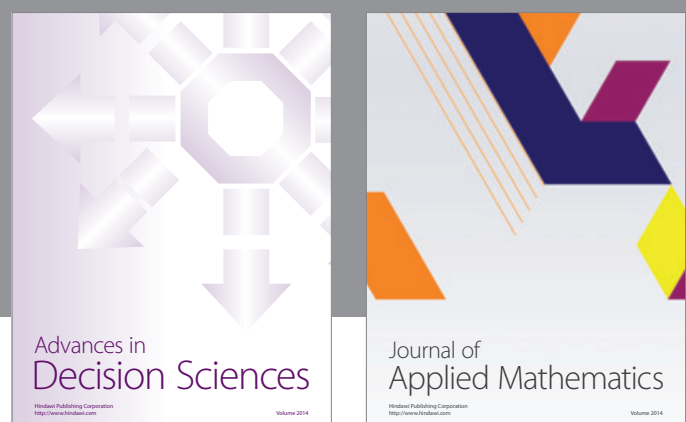

Journal of

Applied Mathematics
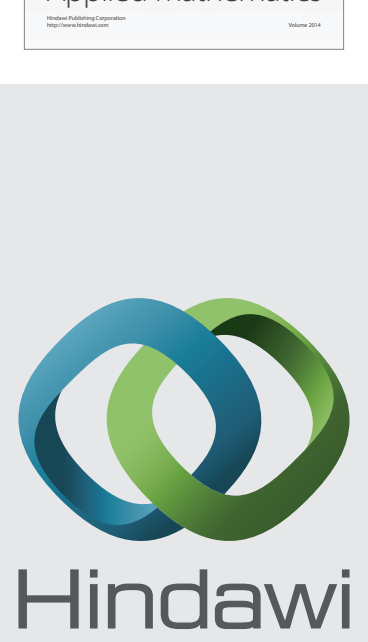

Submit your manuscripts at http://www.hindawi.com
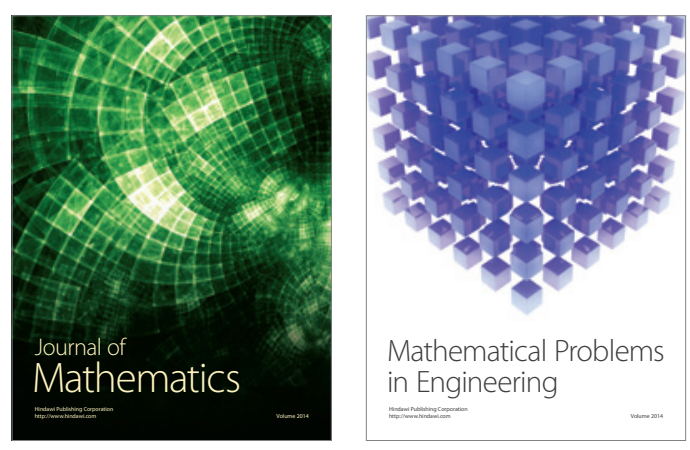

Mathematical Problems in Engineering
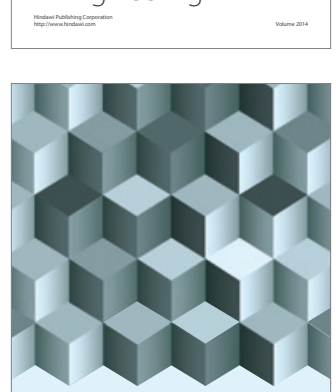

Journal of

Function Spaces
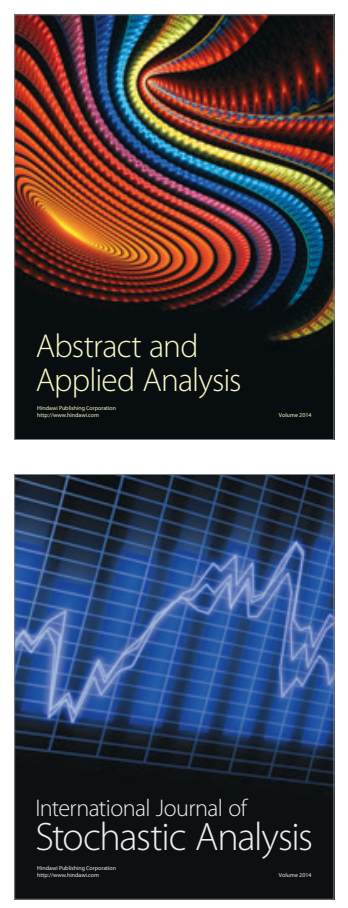

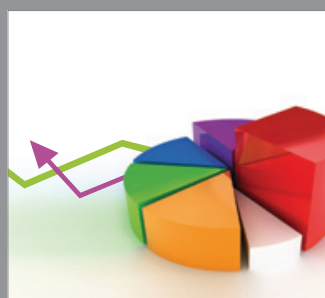

ournal of

Probability and Statistics

Promensencen
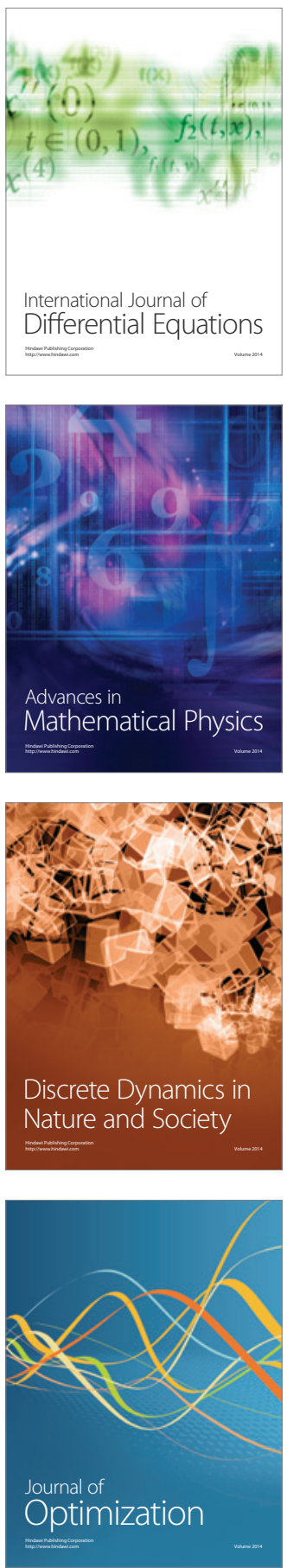